\title{
CONOCIMIENTOS DE MEDICINA BASADA EN EVIDENCIA EN PROFESIONALES DE LA MEDICINA
}

\author{
Knowledge of medical professionals on evidence - based medicine \\ *Carlos Amílcar Fuentes Romero, **Mario Roberto Rodríguez
}

\section{RESUMEN}

La medicina basada en evidencia (MBE) representa un nuevo modelo de investigación y en la práctica médica es sinónimo de actualización. Esta práctica permite identificar la evidencia obteniendo resultados óptimos en la atención de pacientes. Objetivo: Determinar el conocimiento sobre el significado de medicina basada en evidencia y la forma correcta de aplicarla por parte de los profesionales de la medicina. Se aplicaron entrevistas en el Congreso Médico Nacional realizado en San Pedro Sula en el 2011. Metodos: Estudio descriptivo, de corte transversal sobre los conocimientos de los profesionales de la medicina en el tema de MBE previo consentimiento informado para la aplicación de la entrevista. Se entrevistaron un total de 428 médicos aprovechando la realización del Congreso Médico Nacional en la Ciudad de San Pedro Sula en Agosto del 2011. Resultados: La gran mayoría de los entrevistados no conocen el concepto de medicina basada en evidencia y no la aplicarán a su práctica clínica diaria. Conclusiones: El conocimiento sobre MBE del profesional de la medicina, es prácticamente nulo. Se evidencia la necesidad de formar desde estudiante al profesional de la medicina y a

*Profesor de la Escuela Universitaria de las Ciencias de la Salud de la Universidad Nacional Autónoma de Honduras en el Valle de Sula. EUCS/UNAH-VS, Médico Salubrista

** Medico general egresado de Universidad Autónoma de Honduras en el Valle de Sula UNAH-VS

Dirigir correspondencia a: cafr_5993@yahoo.com

Recibido: 17 de Noviembre 2,014 Aprobado 12 de Marzo 2,015 profesores de pregrado en las escuelas de formación de recursos médicos.

\section{PALABRAS CLAVE}

Atención médica, investigación, medicina basada en evidencia.

\section{ABSTRACT}

The evidence-based medicine (EBM) represents a new model of research and medical practice is synonymous update. This practice allows to identify the evidence obtained excellent results in patient care. To determine the knowledge about the significance of evidence-based medicine and the correct way to apply by medical professionals. Interviews were administered in the National Medical Congress held in San Pedro Sula in 2011. Methods: A descriptive, cross-sectional on the expertise of medical professionals on the issue of informed consent MBE for the implementation of the interview. A total of 428 physicians taking the realization of the National Medical Congress in the city of San Pedro Sula in August 2011. We interviewed Results: The majority of respondents did not know the concept of evidence-based medicine and do not apply to your practice daily clinic. Conclusions: Knowledge about MBE medical professional, is practically nil. The need for training is evident from student to professional medical and undergraduate teachers in schools training of medical personnel. 


\section{KEY WORDS}

medical care, research, evidence- based medicine.

\section{INTRODUCCIÓN}

La Medicina Basada en Evidencia (MBE) es un nuevo paradigma en la investigación y la práctica médica. Se usa a menudo como sinónimo de actualización. El profesional de la medicina al ser interrogado dice conocerla y practicarla efectivamente. Sin embargo, la gran mayoría de los profesionales en realidad desconocen el significado. Las definiciones de MBE son muchas y algunas un tanto prolijas. Corresponde al uso consciente, explicito, racional y juiciosa, de la mejor evidencia actual en la toma de decisiones sobre el cuidado sanitario de pacientes, relacionados con la experiencia clínica y los valores de la persona. ${ }^{(1)}$ En este punto, la denominación, racional y juicioso, no significa que por que esta publicado, ya es una verdad, debemos valorar esa evidencia, ponerla en práctica, y es hasta entonces que decidiremos aplicarla a la práctica cotidiana. ${ }^{(2)}$ Con el presente estudio se pretendió, determinar el conocimiento sobre el significado de medicina basada en evidencia, así como la forma correcta de aplicarla, por parte de los profesionales de la medicina. Se aplicaron las entrevistas en el Congreso Medico Nacional realizado en San Pedro Sula en el 2011. Los datos se obtuvieron mediante un cuestionario abierto, previa autorización para su aplicación, por el profesional médico.

\section{MATERIALES Y MÉTODOS}

El estudio correspondió a uno de tipo descriptivo, de corte transversal, sobre el conocimiento de Medicina Basada en Evidencia, entre los médicos que asistieron al Congreso Medico Nacional, realizado en San Pedro Sula en el 2011.

El total de entrevistados fue de 428 profesionales médicos sin distinción de edad, género, procedencia, lugar de trabajo, estudios realizados. Se les aplicó un cuestionario con preguntas abiertas y algunas con posibilidades de respuesta previo consentimiento informado para la aplicación de esta. Los resultados generados dieron respuesta a los objetivos planteados antes de realizar la investigación. Aproximadamente dos tercios de los encuestados correspondió a médicos generales. La gran mayoría entre el rango de edad de 41 a 50 años. Y más de la mitad laborando para instituciones públicas. La distribución en cuanto a género fue equitativa. Se determinó que el profesional de la medicina no entiende el concepto de MBE por lo tanto no puede utilizarla.

\section{RESULTADOS}

Se entrevistaron un total de 428 médicos. En cuanto al rango de edad más frecuente en orden decreciente correspondió a: 156 médicos (36\%) entre los 41-50 años de edad, 151 entre los 31-40 años (35\%), 69 (16\%) menores de 30 años, 48 (11\%) entre 51-60 años y 4 (1\%) de 61 o más años; de los 299 (70\%) eran médicos generales y 129 (30\%) especialistas. Según el género la distribución fue equitativa: 213 hombres y 215 mujeres. Un total de 181 (42.2\%) de los entrevistados laboran para la Secretaria de Salud.

(Tabla No.1) 
Tabla N¹:

Lugar de trabajo

$\begin{array}{lll}\text { Lugar de trabajo } & \text { Frecuencia } & \text { Porcentaje } \\ \text { Secretaria de Salud } & 181 & 42.2 \% \\ \text { I.H.S.S. } & 63 & 14.7 \% \\ \text { Privado } & 170 & 39.7 \% \\ \text { Otro } & 14 & 3.2 \% \\ \text { TOTAL } & \mathbf{4 2 8} & 100 \%\end{array}$

Fuente: Instrumento conocimientos MBE. 2011

Al preguntarles sobre el tiempo que dedicaban para la búsqueda de información sobre sus pacientes 273 (64\%) respondieron que dedicaban una (1) hora al día, 86 (20\%) dedicaban una hora cada dos días, (Tabla No. 2) Hasta aquí, sin identificar la fuente para la búsqueda de la información. Un $72.6 \%$ respondieron que los aspectos relacionados con el tratamiento son los que más les interesan, seguidos en orden de importancia por el diagnóstico, etiología y en última instancia por el pronóstico.

(Tabla No.3)

\section{Tabla N²:}

Tiempo dedicado a la búsqueda de información sobre pacientes

$\begin{array}{lll}\text { Tiempo } & \text { Frecuencia } & \text { Porcentaje } \\ 1 \text { hora al día } & 273 & 64 \% \\ \text { 1 hora cada 2 días } & 86 & 20 \% \\ \text { 1 hora a la semana } & 56 & 13 \% \\ \text { 30 minutos a la semana } & 10 & 2 \% \\ \text { No lo hace } & 3 & 1 \% \\ \text { TOTAL } & 428 & 100 \%\end{array}$

Fuente: Instrumento conocimientos MBE. 2011
Tabla N³:

Tópicos de interés consultados por los médicos

$\begin{array}{ll}\text { Tópico } & \text { Porcentaje } \\ \text { Tratamiento } & 72.6 \% \\ \text { Diagnóstico } & 12.3 \% \\ \text { Etiología } & 10.1 \% \\ \text { Pronóstico } & 5 \% \\ \text { TOTAL } & 100 \% \\ \text { Fuente: Instrumento conocimientos MBE. } 2011\end{array}$

De los entrevistados: 269 (62.8\%) utilizaban los libros de texto como fuente de actualización sobre sus pacientes, 150 (35\%) las revistas médicas y 7 (1.6\%) usaban la red para tal fin (sin especificar que fuentes). 318 (74.2\%) del total respondieron tener conocimientos sobre MBE y 110 (25.8\%) no tenían conocimiento al respecto. En cuanto a la comprensión del significado de MBE, 308 (72\%) refirieron no comprenderlo. Ver Tabla No. 4

Tabla N4:

Comprensión del significado de medicina basada en evidencia

\begin{tabular}{lcc} 
Comprensión & Frecuencia & Porcentaje \\
significado MBE & & \\
SI & 120 & $28 \%$ \\
NO & 308 & $72 \%$ \\
TOTAL & 428 & $100 \%$ \\
\multicolumn{2}{c}{ FUENTE: Instrumento conocimientos MBE. 2011}
\end{tabular}

Un total de $150(35 \%)$, consideraban a la educación médica continua como excelente para la actualización médica, 86 (20\%) le otorgaron la calificación de muy buena, 60 (14\%) la consideraban anticuada, 56 $(12.3 \%)$ ineficaz, $47(11 \%)$ regular y 29 (7\%) mala. 
Pero aun así, 360 (84.3\%) respondieron que la MBE tiene una calificación de buena a excelente como medio para la actualización de los profesionales de la medicina.

Al pedirles que expresaran un concepto sobre MBE, solo $1(0.2 \%)$ del total de entrevistados respondió que es el uso juicioso de la mejor evidencia para aplicarla al cuidado de los pacientes. El resto tiene un concepto poco claro o errado.

\section{DISCUSIÓN}

La Medicina Basada en Evidencia (MBE) es en la actualidad una tendencia en el trabajo de los médicos y es de gran difusión, lo que ha provocado cambios en la práctica clínica. Esto se evidencia con los artículos que se han escrito sobre el tema. Ahora bien ¿Cuáles son las razones para esta difusión del tema? Son muchas. Al tratar de enumerarlas nos encontramos con las siguientes:

1) La necesidad diaria de obtener información fidedigna sobre la enfermedad de nuestros pacientes (tratamiento, diagnóstico, etiología, pronóstico), se ha manifestado que un profesional de la medicina necesita obtenerla al menos cinco veces por cada paciente hospitalizado y dos veces por cada tres pacientes atendidos ambulatoriamente. ${ }^{(3)}$ Algunos han llegado a decir que esta podría afectar hasta ocho decisiones clínicas por día. 2) Otra razón corresponde, que a diario el médico necesita respuestas sobre lo que aqueja a sus pacientes, históricamente esta información se encontraba en revistas, algunas variable o, en libros de texto casi siempre desactualizados. ${ }^{(4)}$ Se menciona como causa la gran cantidad de información publicada y el poco tiempo para buscarla. ${ }^{(4,5)}$ 3) Y por último, el desconocimiento casi total sobre el significado de lo que en realidad es la medicina basada en evidencia, por los profesionales médicos. En un estudio realizado en un servicio de neumología en un Hospital de Managua, en el 2010 se le preguntó al personal médico ¿cuáles eran los tópicos de interés al buscar información sobre sus pacientes? El $71 \%$ de los encuestados respondió que requieren información sobre el tratamiento, un $21 \%$ sobre el pronóstico y $8 \%$ sobre el diagnóstico. ${ }^{(6)}$ En nuestro estudio de 428 entrevistados, el $72.6 \%$ respondió su interés de conocer sobre aspectos del tratamiento de los pacientes, seguido de un $12.3 \%$ sobre el diagnóstico y, menos frecuentes los aspectos relacionados con la etiología y pronóstico. Esto relacionado con los avances de la terapéutica médica qué bien sabemos día a día los progresos en ese campo son importantes. Antes surgir la era de la informática conseguir la información era dificultoso ya que los artículos seleccionados no estaban en las revistas en las hemerotecas de nuestros países. Así que, la MBE ha venido a replantear la manera que transformamos la información en interrogantes sobre el diagnóstico, tratamiento y pronóstico de los padecimientos de nuestros pacientes optimizando la información en un mundo tan globalizado como en el que vivimos. Antes el tiempo dedicado a la búsqueda de información sobre los pacientes era reducido pero con la tecnología de la información y del conocimiento (TICS) es diferente. Hoy se dice que un profesional de la medicina debe leer al menos, veinte artículos por día, para decir que esta actualizado.(4) Pero, no solo se trata de leerlos, debe valorarlos y más aún aplicarlos a su práctica cotidiana. Para evaluarlos se usara el nivel de evidencia y 
el grado de recomendación prefiriendo aquellos estudios que estén en nivel de evidencia I o II y grado de recomendación A o B. En especial los estudios clínicos controlados aleatorizados y los de cohorte..$^{(7-10)}$

Haciendo un análisis observamos que el tiempo dedicado por los profesionales médicos a la búsqueda y análisis de información sobre sus pacientes es poco por lo que se ha determinado que el médico una vez graduado, pierde el interés en la actualizacizón. La educación médica continua está demostrado que no produce una mejora sustancial en el manejo de pacientes. ${ }^{(5)}$

Hace unos años se realizó un estudio en los Estados Unidos para determinar los factores que afectan el grado de conocimiento base de médicos especialistas en medicina interna. Se aplicó un cuestionario que tenían entre 5 y 15 años de haberse graduado. Encontrando una correlación inversa entre los puntaje obtenidos y los años desde su graduación. Como conclusión se determinó que, un médico por cada año posterior a su graduación sin actualización pierde un 10\% de los conocimientos adquiridos en su formación. ${ }^{11)}$

Aunque más del $80 \%$ apenas dedican una hora para la búsqueda de información sobre sus pacientes y más del 90\%, utiliza libros de texto y revistas para tal fin en bibliografía desactualizada y desestimable en su validez. Además, no tienen una idea clara del significado de MBE, no pueden acceder a la misma ya que no saben que fuentes consultar. Y más aun, no tienen idea alguna de lo que es MBE, por increíble que parezca, solo una persona de las entrevistadas (0.6\%) respondió con claridad el concepto. La MBE ha venido a establecer un punto de unión entre la "mejor evidencia" y la práctica médica cotidiana ofreciendo a los pacientes una atención de calidad haciendo que los valores y preferencias de nuestros pacientes sean lo más importante en el accionar del médico. Esto permite una participación más activa de los pacientes en la toma de decisión ${ }^{(5)}$ Recordemos además que, la sola evidencia no es suficiente en la toma de una decisión, debemos evaluar adecuadamente al paciente, establecer un diagnóstico correcto, establecer el riesgo/beneficio de una acción, los costos de la acción y sobre todo las preferencias del paciente. A este ciclo se le conoce como el ciclo de la MBE. ${ }^{(4,12)}$

La MBE representa un excelente medio para la actualización de los profesionales de la medicina. Debemos aprenderla, y aplicarla a nuestra práctica diaria. Profundizar en esta corriente de pensamiento medico, acordes con el Siglo XXI.

\section{BIBLIOGRAFÍA}

1. Guyatt G, Rennier D. User's guide to the medical literature, a manual for evidence based practice. Chicago. American Medical Association. 2002.
2. Strauss S, Richardson S, Glasziou P, Haynes B. Evidence based medicine. How to practice and teach. 3rd.ed. Churchill Livingstone. Philadelphia. 2005. 
3. Covell DG, Umann GC, Manning PR. Information needs in office practice: are they being met? Ann Int Med. 1985; 103: 596-9.

4. Málaga Rodríguez G, Sánchez Mejía A. Medicina basada en evidencia: aportes a la práctica médica actual y dificultades para su implementación. Rev Med Hered. 2009; 20(2): 103-7.

5. Principios de medicina basada en evidencia. Centro de Investigaciones y Estudios de la Salud. Universidad Nacional Autónoma de Nicaragua-Managua. 2009.

6. Encuesta sobre el interés de información sobre pacientes en Servicio de Neumología Hospital Fernando Vélez Paiz. Centro de Investigaciones y Estudios de la Salud. Universidad Nacional Autónoma de Nicaragua. Managua. 2010.

7. Marzo Castillejo M, Viana Zulaica C. Calidad de la evidencia y grado de recomendación. Guías de práctica clínica: Series MBE. 2007; 7: (Sulemento)1-6.
8. Levels of evidence. Oxford Centre for Evidence Medicine Based. 2011. (disponible en: http://cebm.net/2011oxford-cebm-levels-evidence-introduction-document/).

9. Levels of evidence. Essential Evidence Plus. 2011. (disponible en: http://essentialevidenceplus.com/product/ebm_loe.cfm?show=oxford).

10. Levels of evidence and grades of recommendations. Healt Sciences Libraries. University of Minnesota. 2014. November.16.

(disponible en: http://hs.lib.umn.edu/bio$\mathrm{med} / \mathrm{help}$ /levels-evidence-and-gradesrecommendations)

11. Ramsey PG, Carline JD, Inui TS, Larson EB, LoGerfo JP, Norcini JJ, Wenrich MD. Changes over time in the knowledge base of practicing internist. JAMA. 1991; 266(8): 1103-1107.

12. Gonzales Guitián C. Medicina basada en evidencia en internet: como buscar la mejor evidencia científica. Hospital Juan Canalejo. Universidad A. Coruña. 2009 